\title{
Convenient syntheses of new quinoline nucleosides bearing amino acid esters
}

\author{
Ibrahim A. I. Ali \\ Department of Chemistry, Faculty of Science, Suez Canal University, Ismailia, Egypt \\ E.mail:ibrahim3369@yahoo.com
}

Dedicated to Prof. Ezz El-Din M. S. Salem for his $\mathbf{7 0}^{\text {th }}$ birthday

\begin{abstract}
Quinoline reverse nucleosides 4, 5 were prepared by reaction of quinolines 1, 2 with methyl 2,3$O$-isopropylidene-5- $O-p$-toluenesulfonyl- $\beta$-D-ribofuranoside (3) in the presence of sodium hydride. Quinoline nucleosides bearing an amino acid ester residue 12-16 were prepared by azide coupling method from ester 4 . The synthesized compounds were characterized by elemental analysis, MALDI MS and NMR data.
\end{abstract}

Keywords: Nucleosides, quinoline, amino acids and dipeptide, azide coupling, ribose-series

\section{Introduction}

The synthesis of quinoline and its derivatives have attracted considerable attention of organic and medicinal chemists for many years. ${ }^{1-4}$ The structural core of quinoline is frequently associated with medicinal applications, such as anti-cancer, ${ }^{5}$ antimicrobial, ${ }^{6}$ HIV-1 integrase inhibitors, ${ }^{7}$ HIV protease inhibitors ${ }^{8}$ antileishmanial activity, ${ }^{9}$ NK-3 receptor antagonists ${ }^{10}$ and pLT antagonists. ${ }^{11-13}$

Quinoline carboxamides are bioisosteres of the NK-3 antagonist SB 218795 and were reported to possess local anesthetic, potent cholinesterase inhibitor activities ${ }^{14}$ and potent and orally active PDE4 inhibitor. ${ }^{15}$

The intensive efforts to find effective therapeutic agents with antiviral and antitumor activities have directed many researchers to synthesize a series of modified nucleosides or their analogues. Among the enormous number of published articles on modified heterocyclic nucleosides there are only a few publications that report the amino acid coupling of heterocyclic nucleosides. ${ }^{16-18}$ 
This type of modified amino acid nucleosides brings the potential of simultaneous binding and recognition of two complementary structure residues; amino acid and heterocyclic ring, which might interact with the poly nucleotides of the receptor recognition sites.

The aim of the present study was to synthesize a series of quinoline nucleosides in the riboseries substituted at position 4 by a spacer linked with a series of amino acids and dipeptides carboxamides as potential chemotherapeutic agents.

\section{Result and Discussion}

In view of these facts and in continuation of our efforts in synthesizing various bioactive molecules, ${ }^{19-23}$ we have found it desirable to synthesize a series of quinoline nucleoside bearing amino acid methyl esters and dipeptides at position 4.

Treatment of quinoline $\mathbf{1}$ and $\mathbf{2}^{24}$ with protected ribose tosylate $\mathbf{3}$ in the presence of $\mathrm{NaH}$ in dry DMF afforded methyl 1-(methyl 5-deoxy-2,3-O-isopropylidene- $\beta$-D-ribofuranosyl-5-yl)-2oxo-1,2-dihydroquinoline-4-carboxylate (4) and acid 5, respectively in $85-90 \%$ yields according to a slightly modified procedure by the displacement of the tosyloxy group. ${ }^{25,26}$

The structure of reverse nucleoside $\mathbf{5}$ was chemically confirmed by an equivocal synthesis from the ester derivative 4 by hydrolysis in aqueous alcoholic $\mathrm{KOH}$. On the other hand, the attempted esterification of $\mathbf{5}$ with thionyl chloride in absolute methanol afforded fully deprotected "reversed" nucleoside ester $\mathbf{8}$ in $60 \%$ yield. The subsequent acetylation of $\mathbf{8}$ gave diacetyl derivative 9 (Scheme 1).

Carboxamides play a key role in medicinal chemistry. They are neutral, stable, have both hydrogen bond donating and accepting properties necessary for biological molecule recognition. $^{27}$

Both ester 4 and acid 5 are excellent key intermediates for the simple chemical modification of the quinoline skeleton to produce a carboxamide moiety at position 4 . The ester 4 was boiled with hydrazine hydrate in ethyl alcohol to afford the hydrazide 6, which was subsequently converted into azide 7 by treatment with $\mathrm{NaNO}_{2}$ and $\mathrm{HCl}$ mixture. 


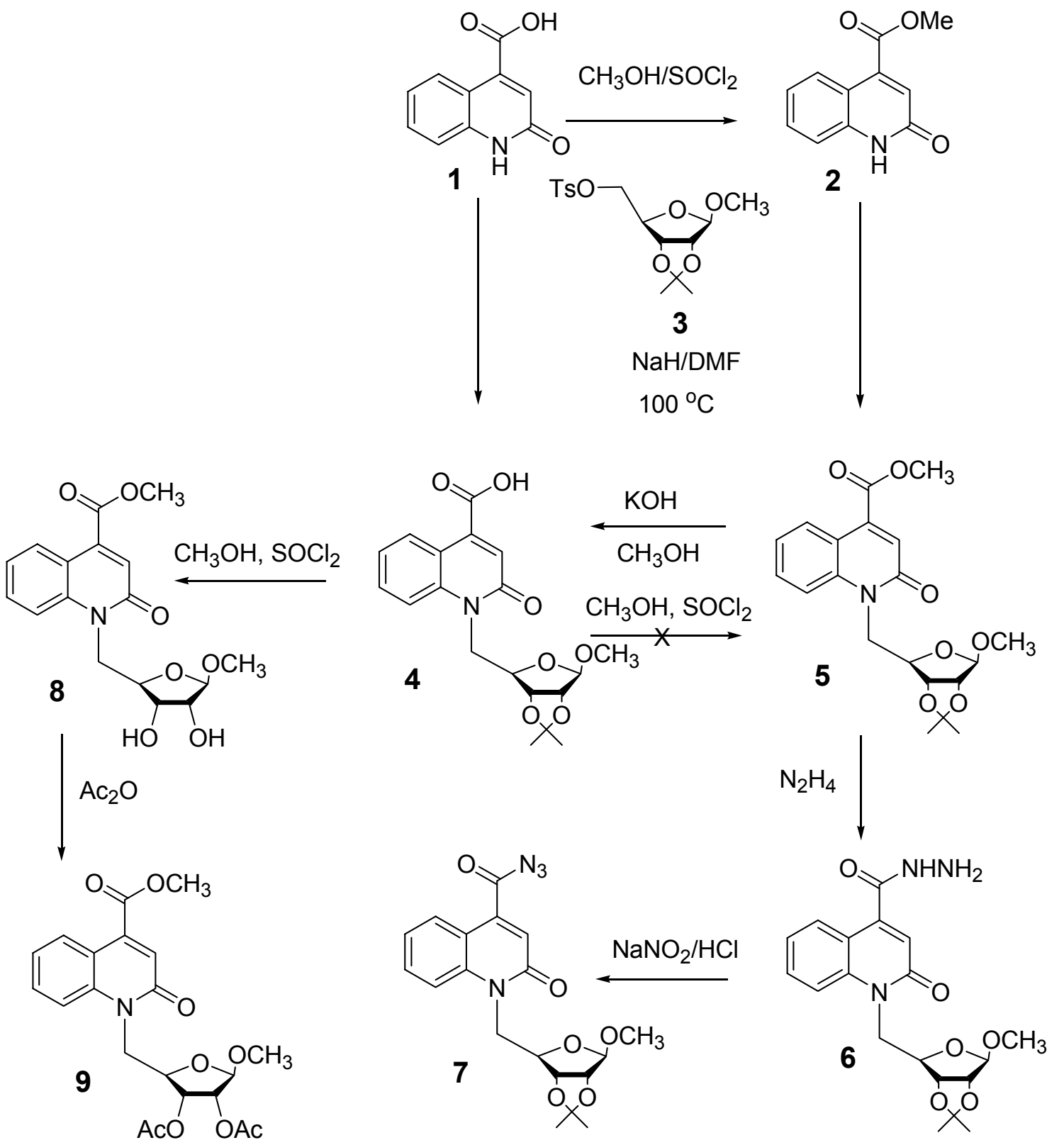

\section{Scheme 1}

The synthesis of the target carboxamide 10 and amino acid derivatives 12a-e, 14 was efficiently performed from key intermediate ester $\mathbf{4}$ via the azide coupling method, ${ }^{22,}{ }^{28}$ which was reported to minimize the degree of racemization in amino acid coupling. The azide 7 reacted with piperidine in ethyl acetate to give the corresponding 1-(methyl 5-deoxy-2,3-Oisopropylidene- $\beta$-D-ribofuranosyl-5-yl)-2-oxo-4-(piperidine-1-carbonyl) 1,2-dihydroquinoline (10) in $77 \%$ yield.

Similarly, the azide 7 reacted with amino acid methyl ester hydrochloride 11a-e (glycine, Lalanine, L-valine, L-leucine, L-methionine) and $\mathbf{1 3}$ in the presence of triethyl amine in ethyl 
acetate to afford methyl-2-\{[1-(methyl 5-deoxy-2,3-O-isopropylidene- $\beta$-D-ribofuranosyl-5-yl)]2-oxo-1,2-dihydroquinoline-4-carboxamido $\}$ alkanoate 12a-e and $\mathbf{1 4}$ in good yield.
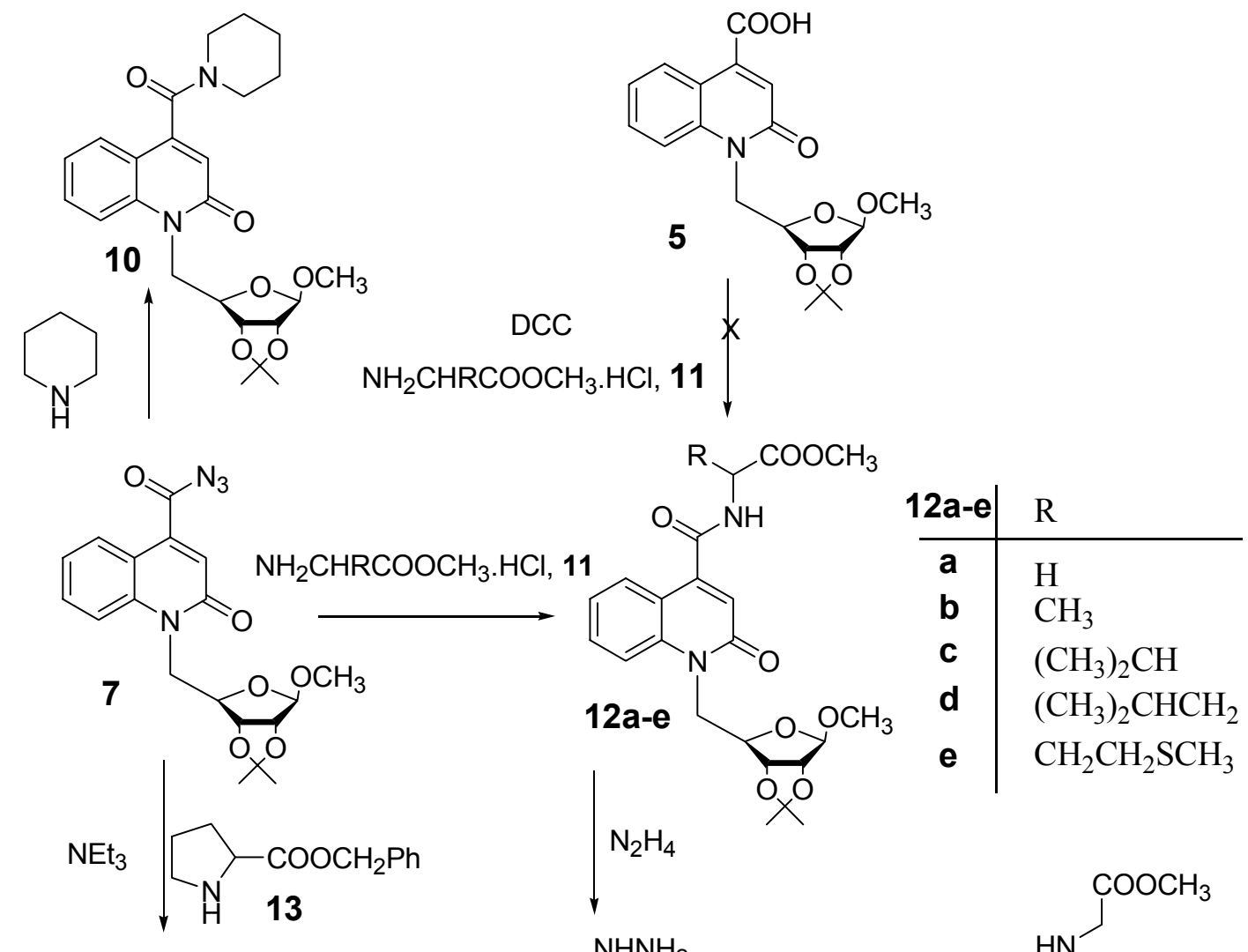<smiles>COC1OC2(COc3ccccc3)OCC1OC2Cn1c(=O)cc(C(=O)N2CCCC2C(=O)OCc2ccccc2)c2ccccc21</smiles><smiles>CO[C@H]1O[C@@H]2COC1(C)O2</smiles><smiles>COCC12CC(Cn3c(=O)cc(C(=O)NCC(=O)NCC(=O)O)c4ccccc43)OC(C)(O1)C(OC)O2</smiles>

\section{Scheme 2}

The DCC coupling is one of the major tools employed in literature to introduce peptide bonds by the reaction of acid with amino acid methyl ester. The synthesis of the target amino acid derivatives 12a-e from the corresponding acid 5 via DCC coupling could be valuable for chemical structure confirmation. However, the reaction of acid $\mathbf{5}$ with glycine methyl ester hydrochloride 11a in the presence of DCC, HOBt and triethyl amine failed to give the desired product 12a. 
Further development of azide coupling was obtained by the synthesis of the dipeptide derivative 16. Hydrazinolysis of the amino acid ester 12a (Gly) afforded the hydrazide 15. Nitrosation of hydrazide $\mathbf{1 5}$ gave the azide. The in situ generated azide solution in ethyl acetate reacted with glycine methyl ester hydrochloride 11a, in the presence of triethyl amine to afford the dipeptide 16 (Scheme 2).

The structure assignment of the amino acid esters 12a-e and $\mathbf{1 6}$ is based on spectral analysis, (Figure 1). The ${ }^{1} \mathrm{H}$ NMR spectrum of the glycine derivative 12a exhibits three singlet signals at $\delta$ 4.99, 3.75 and $3.31 \mathrm{ppm}$ corresponding to $\mathrm{H}-1$ and $2 \mathrm{OCH}_{3}$ respectively. The ${ }^{13} \mathrm{C}$ NMR spectrum of 12a shows $107.8,54.9,41.5 \mathrm{ppm}$ attributed to $(\mathrm{C}-1), \mathrm{OCH}_{3}$ and $\mathrm{NCH}_{2}$ respectively. The ${ }^{1} \mathrm{H}$ NMR for all amino acid derivatives 12b (Ala), 12c (Val), 12d (Leu), 12e (Met), gives triplet or doublet centered at $\delta 6.60 \mathrm{ppm}$ attributed to $\mathrm{NH}$.

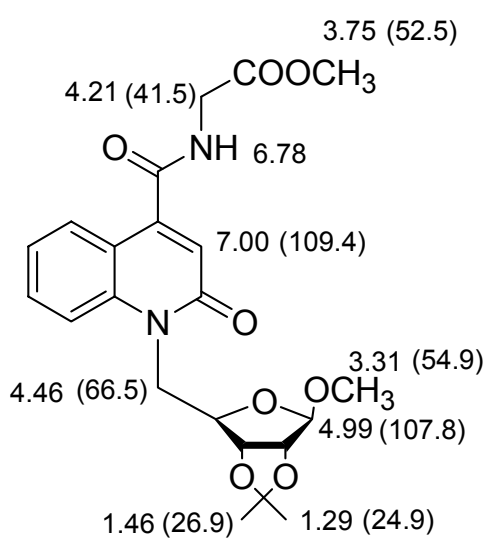

$12 a$

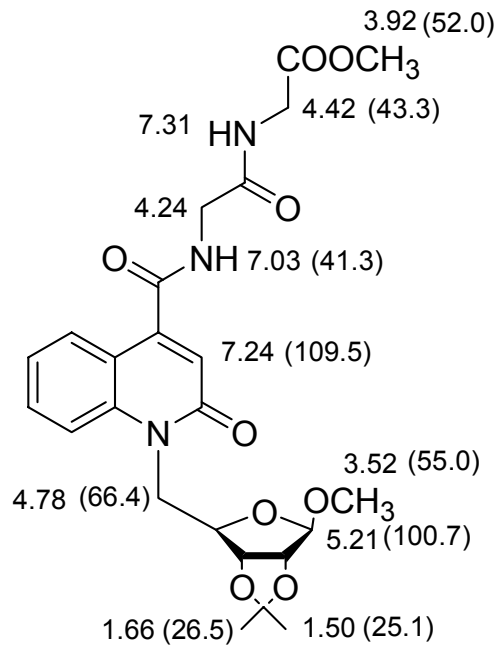

16

Figure 1. Selected ${ }^{1} \mathrm{H}$ NMR and ${ }^{13} \mathrm{C}$ NMR spectral data of amino acid derivative 12a and dipeptide 16.

\section{Conclusions}

In conclusion, an efficient and very simple method for the syntheses of various quinoline reverse nucleosides bearing an amino acid ester residue 12-16 were prepared by azide coupling method from ester $\mathbf{4}$ in high yields as potential chemotherapeutic agents.

\section{Experimental Section}

General Procedures. Solvents were purified and dried in the usual way. The boiling range of the petroleum ether used was $40-60^{\circ} \mathrm{C}$. Thin layer chromatography (TLC): silica gel $60 \mathrm{~F}_{254}$ 
plastic plates (E. Merck, layer thickness $0.2 \mathrm{~mm}$ ) detected by UV absorption. Melting points were determined on a Buchi 510 melting-point apparatus and the values are uncorrected. NMR spectra measured with Bruker AC 250 (250 MHz). TMS (0.00 ppm) as internal standard. MALDI-MS were measured with a KRATOS Analytical Compact, using 2,5 dihydroxybenzoic acid $(\mathrm{DHB})$ as matrix. The $(\mathrm{M}+\mathrm{Na})^{+}$and $(\mathrm{M}+\mathrm{K})^{+}$ions were peak matched using ions derived from the 2,5-dihydroxybenzoic acid matrix. Elemental analyses were performed on a Flash EA1112 instrument at the Microanalytical laboratory, Faculty of Science, Suez Canal University, Ismailia, Egypt. The starting compounds $\mathbf{1}, \mathbf{2}^{26}$ and $\mathbf{3}^{24,25}$ were prepared according to the method described.

\section{Preparation of compound 4}

A mixture of quinoline $1(2.0 \mathrm{~g}, 10.0 \mathrm{mmol})$ and $\mathrm{NaH}(0.24 \mathrm{~g}, 10.0 \mathrm{mmol})$ in dry $\mathrm{DMF}(50 \mathrm{ml})$ was stirred at $100^{\circ} \mathrm{C}$ for $1 \mathrm{~h}$. The sugar derivative $3(3.42 \mathrm{~g}, 10.0 \mathrm{mmol})$ was added and the mixture was stirred at $100^{\circ} \mathrm{C}$ for $5 \mathrm{~h}$. The solution was evaporated to dryness. The residue was purified by column chromatography petroleum ether/ethyl acetate $(8: 1)$ as eluent to give 4 .

Methyl 1-(methyl 5-deoxy-2,3-O-isopropylidene- $\beta$-D-ribofuranosyl-5-yl)-2-oxo-1,2dihydroquinoline-4-carboxylate (4). White powder $(3.50 \mathrm{~g}, 90 \%)$; mp 156-157 ${ }^{\circ} \mathrm{C} .{ }^{1} \mathrm{H}$ NMR $\left(250 \mathrm{MHz}, \mathrm{CDCl}_{3}\right): \delta 8.51(1 \mathrm{H}, \mathrm{d}, J=7.7 \mathrm{~Hz}, \mathrm{ArH}) ; 7.75(1 \mathrm{H}, \mathrm{d}, J=7.7 \mathrm{~Hz}, \mathrm{ArH}) ; 7.62-7.58$ $(3 \mathrm{H}, \mathrm{m}, \mathrm{ArH}, \mathrm{CH}) ; 4.97(1 \mathrm{H}, \mathrm{s}, \mathrm{H}-1) ; 4.76(1 \mathrm{H}, \mathrm{d}, J=5.9 \mathrm{~Hz}, \mathrm{H}-2) ; 4.61(1 \mathrm{H}, \mathrm{d}, J=5.9 \mathrm{~Hz}, \mathrm{H}-$ 3); 4.52-4.47 (1H, m, H-4); 4.43-4.37 (2H, m, H-5, H-5 ); $3.88\left(3 \mathrm{H}, \mathrm{s}, \mathrm{OCH}_{3}\right) ; 3.28(3 \mathrm{H}, \mathrm{s}$, $\left.\mathrm{OCH}_{3}\right) ; 1.44\left(3 \mathrm{H}, \mathrm{s}, \mathrm{CH}_{3}\right) ; 1.27\left(3 \mathrm{H}, \mathrm{s}, \mathrm{CH}_{3}\right) .{ }^{13} \mathrm{C} \mathrm{NMR}\left(62.5 \mathrm{MHz}, \mathrm{CDCl}_{3}\right): \delta 166.0 ; 160.0$ (2CO); 147.3; 137.9; 129.8; 127.7; 125.5; 125.3; 122.0; $115.2(\mathrm{Ar}-\mathrm{C}) ; 112.6\left(\mathrm{CMe}_{2}\right) ; 109.4(\mathrm{C}-$ 1); 85.2 (C-2); 84.4 (C-3); 82.0 (C-4); $66.3(\mathrm{C}-5) ; 54.8 ; 52.5\left(2 \mathrm{OCH}_{3}\right) ; 26.4 ; 24.9\left(2 \mathrm{CH}_{3}\right)$. (MALDI, positive mode, matrix: DHB): $\mathrm{m} / \mathrm{z}=388.8(\mathrm{M})^{+} ; 411.3(\mathrm{M}+\mathrm{Na})^{+}$. Anal. Calcd. For $\mathrm{C}_{20} \mathrm{H}_{23} \mathrm{NO}_{7}$ (389.40): C, $61.69 \%, \mathrm{H}, 5.95 \%$, N, $3.60 \%$; found $\mathrm{C}, 61.42 \%, \mathrm{H}, 5.88 \%$, N, 3.42 $\%$.

\section{Preparation of compound 5}

Method A. A mixture of quinoline $2(1.90 \mathrm{~g}, 10.0 \mathrm{mmol})$ and $\mathrm{NaH}(0.48 \mathrm{~g}, 20.0 \mathrm{mmol})$ in dry DMF $(50 \mathrm{ml})$ was stirred at $100^{\circ} \mathrm{C}$ for $1 \mathrm{~h}$. The sugar derivative $3(3.42 \mathrm{~g}, 10.0 \mathrm{mmol})$ was added and the mixture was stirred at $100^{\circ} \mathrm{C}$ for $5 \mathrm{~h}$. The solution was evaporated to dryness and the residue was acidified by dil $\mathrm{HCl}$ and extracted with $\mathrm{CHCl}_{3}(100 \mathrm{~mL})$. The combind organic extracts were dried over $\mathrm{Na}_{2} \mathrm{SO}_{4}$, filtered and evaporated to dryness and the residue was chromatographed using petroleum ether/ethyl acetate (6:1) as eluent.

1-(Methyl 5-deoxy-2,3-O-isopropylidene- $\beta$-D-ribofuranosyl-5-yl)-2-oxo-1,2-dihydroquinoline-4-carboxylic acid (5). White crystals (3.20 g, $85 \%)$; mp 212-213 ${ }^{\circ} \mathrm{C} .{ }^{1} \mathrm{H}$ NMR $(250$ $\left.\mathrm{MHz}, \mathrm{CDCl}_{3}\right): \delta 12.83(1 \mathrm{H}, \mathrm{bs}, \mathrm{OH}) ; 8.36(1 \mathrm{H}, \mathrm{d}, J=8.2 \mathrm{~Hz}, \mathrm{ArH}) ; 7.60-7.45(2 \mathrm{H}, \mathrm{m}, \operatorname{ArH})$; 7.28-7.19 (2H, m, ArH, CH); $5.01(1 \mathrm{H}, \mathrm{s}, \mathrm{H}-1)$; $4.74(1 \mathrm{H}, \mathrm{d}, J=5.8 \mathrm{~Hz}, \mathrm{H}-2) ; 4.63(1 \mathrm{H}, \mathrm{d}, J=$ $5.8 \mathrm{~Hz}, \mathrm{H}-3)$; 4.54-4.49 (1H, m, H-4); 4.44-4.41 (2H, m, H-5, H-5 '); 3.34 (3H, s, $\left.\mathrm{OCH}_{3}\right) ; 1.48$ $\left(3 \mathrm{H}, \mathrm{s}, \mathrm{CH}_{3}\right) ; 1.32\left(3 \mathrm{H}, \mathrm{s}, \mathrm{CH}_{3}\right) .{ }^{13} \mathrm{C} \mathrm{NMR}\left(62.5 \mathrm{MHz}, \mathrm{CDCl}_{3}\right): \delta 164.6,163.5(2 \mathrm{CO}) ; 140.2$; 
138.9; 138.7; 131.2; 126.4; 124.4; 123.4; 112.3 (Ar-C); $112.8\left(\mathrm{CMe}_{2}\right) ; 109.6$ (C-1); 85.2 (C-2); 84.0 (C-3); 81.7 (C-4); $66.1(\mathrm{C}-5) ; 55.1\left(\mathrm{OCH}_{3}\right) ; 26.4 ; 24.9\left(2 \mathrm{CH}_{3}\right)$. (MALDI, positive mode, matrix: DHB): $\mathrm{m} / \mathrm{z}=397.6(\mathrm{M}+\mathrm{Na})^{+}$. Anal. Calcd. For $\mathrm{C}_{19} \mathrm{H}_{21} \mathrm{NO}_{7}$ (375.37): $\mathrm{C}, 60.79 \%, \mathrm{H}$, $5.64 \%, \mathrm{~N}, 3.73 \%$; found $\mathrm{C}, 61.02 \%, \mathrm{H}, 5.35 \%, \mathrm{~N}, 4.11 \%$.

Method B. A solution of $4(1.95 \mathrm{~g}, 5.0 \mathrm{mmol})$ in methanol $(40 \mathrm{~mL})$ and $5 \% \mathrm{KOH}(6 \mathrm{~mL})$ was stirred for $6 \mathrm{~h}$. The solution was evaporated to dryness and the residue was dissolved in water and acidified with dil $\mathrm{HCl}$. The white precipitate was filtered and chromatographed using petroleum ether/ethyl acetate $(6: 1)$ to give 5 (1.20 g, $64 \%)$.

\section{Preparation of hydrazide 6}

To a solution of etser $4(3.90 \mathrm{~g}, 10.0 \mathrm{mmol})$ in methyl alcohol $(50 \mathrm{~mL})$ was added hydrazine hydrate $(2.4 \mathrm{~mL}, 50.0 \mathrm{mmol})$. The reaction mixture was refluxed for 4 hours, cooled and the resultant precipitate was filtered off, washed with ethanol and ether then crystallized from methanol to yield the hydrazide 6 .

1-(Methyl 5-deoxy-2,3- $O$-isopropylidene- $\beta$-D-ribofuranosyl-5-yl)-2-oxo-1,2-dihydroquinoline-4-carboxylic acid hydrazide (6). White crystals $(3.40 \mathrm{~g}, 87 \%)$; mp 197-198 ${ }^{\circ} \mathrm{C} .{ }^{1} \mathrm{H}$ NMR $\left(250 \mathrm{MHz}, \mathrm{CDCl}_{3}\right): \delta 8.05(1 \mathrm{H}, \mathrm{d}, J=7.1 \mathrm{~Hz}, \mathrm{ArH}) ; 7.72(1 \mathrm{H}, \mathrm{d}, J=7.1 \mathrm{~Hz}, \mathrm{ArH}) ; 7.62-$ $7.32(2 \mathrm{H}, \mathrm{m}, \mathrm{ArH}) ; 6.95(1 \mathrm{H}, \mathrm{s}, \mathrm{CH}) ; 4.97(1 \mathrm{H}, \mathrm{s}, \mathrm{H}-1) ; 4.58(1 \mathrm{H}, \mathrm{d}, J=6.0 \mathrm{~Hz}, \mathrm{H}-2)$; 4.56-4.45 (4H, m, H-3, H-4, H-5, H-5 $) ; 3.88\left(3 \mathrm{H}, \mathrm{s}, \mathrm{OCH}_{3}\right) ; 3.20\left(2 \mathrm{H}, \mathrm{bs}, \mathrm{NH}_{2}\right) ; 1.43\left(3 \mathrm{H}, \mathrm{s}, \mathrm{CH}_{3}\right) ; 1.25$ $\left(3 \mathrm{H}, \mathrm{s}, \mathrm{CH}_{3}\right) .{ }^{13} \mathrm{C} \mathrm{NMR}\left(62.5 \mathrm{MHz}, \mathrm{CDCl}_{3}\right): \delta 167.6,160.5(2 \mathrm{CO}) ; 143.2 ; 134.2 ; 130.2 ; 127.7$; 125.1; 121.7; 112.5; 112.1 (Ar-C); $111.4\left(\mathrm{CMe}_{2}\right) ; 109.4$ (C-1); 85.2 (C-2); 84.5 (C-3); 82.0 (C4); $66.3(\mathrm{C}-5) ; 54.9\left(\mathrm{OCH}_{3}\right) ; 26.4 ; 24.9\left(2 \mathrm{CH}_{3}\right)$. Anal. Calcd. For $\mathrm{C}_{19} \mathrm{H}_{23} \mathrm{~N}_{3} \mathrm{O}_{6}(389.40)$ : C, 58.60 $\%, \mathrm{H}, 5.95 \%, \mathrm{~N}, 10.79 \%$; found C, $58.46 \%, \mathrm{H}, 5.81 \%, \mathrm{~N}, 10.92 \%$.

\section{Preparation of azide 7}

To a cold solution $\left(-5^{\circ} \mathrm{C}\right)$ of hydrazide derivative $6(3.89 \mathrm{~g}, 10.0 \mathrm{mmol})$ in HOAc $(10 \mathrm{ml}), 1 \mathrm{~N}$ $\mathrm{HCl}(30 \mathrm{ml})$, and water $(150 \mathrm{ml})$ was added a solution of $\mathrm{NaNO}_{2}(1.0 \mathrm{~g}, 15.0 \mathrm{mmol})$ in cold water $(10 \mathrm{ml})$. After stirring at $-5^{\circ} \mathrm{C}$ for $15 \mathrm{~min}$, the yellow syrup was formed. The azide was taken in cold ethyl acetate $(100 \mathrm{ml})$, washed with cold $3 \% \mathrm{NaHCO}_{3}, \mathrm{H}_{2} \mathrm{O}$ and finally dried $\left(\mathrm{Na}_{2} \mathrm{SO}_{4}\right)$. The solvent was evaporated in vacuo to give the pure azide 7 .

1-(Methyl 5-deoxy-2,3- $O$-isopropylidene- $\beta$-D-ribofuranosyl-5-yl)-2-oxo-1,2-dihydro quinoline-4-carbonyl azide (7). Yellow powder (2.60 g, $65 \%$; mp 68-69 ${ }^{\circ} \mathrm{C} .{ }^{1} \mathrm{H}$ NMR (250 $\left.\mathrm{MHz}, \mathrm{CDCl}_{3}\right): \delta$ 7.89-7.60 (2H, m, ArH); 7.51-7.45 (2H, m, ArH); $6.71(1 \mathrm{H}, \mathrm{s}, \mathrm{CH}) ; 5.03(1 \mathrm{H}, \mathrm{s}$, $\mathrm{H}-1) ; 4.81(1 \mathrm{H}, \mathrm{d}, J=5.8 \mathrm{~Hz}, \mathrm{H}-2) ; 4.65(1 \mathrm{H}, \mathrm{d}, J=5.8 \mathrm{~Hz}, \mathrm{H}-3)$; 4.62-4.50 (3H, m, H-4, H-5, $\left.\left.\mathrm{H}^{\prime}\right)^{\prime}\right) ; 3.34\left(3 \mathrm{H}, \mathrm{s}, \mathrm{OCH}_{3}\right) ; 1.48\left(3 \mathrm{H}, \mathrm{s}, \mathrm{CH}_{3}\right) ; 1.32\left(3 \mathrm{H}, \mathrm{s}, \mathrm{CH}_{3}\right) .{ }^{13} \mathrm{C} \mathrm{NMR}\left(62.5 \mathrm{MHz}, \mathrm{CDCl}_{3}\right)$ : $\delta 178.5,160.6$ (2CO); 144.5; 136.2; 130.6; 127.7; 124.7; 122.3; 115.7 (Ar-C); $111.4\left(\mathrm{CMe}_{2}\right)$; $109.4(\mathrm{CH}) ; 107.8$ (C-1); 85.3 (C-2); 84.5 (C-3); 82.1 (C-4); 66.3 (C-5); $54.9\left(\mathrm{OCH}_{3}\right) ; 26.5,25.0$ $\left(2 \mathrm{CH}_{3}\right)$. (MALDI, positive mode, matrix: DHB): $\mathrm{m} / \mathrm{z}=422.1(\mathrm{M}+\mathrm{Na})^{+}$. Anal. Calcd. For $\mathrm{C}_{19} \mathrm{H}_{20} \mathrm{~N}_{4} \mathrm{O}_{6}$ (400.39): C, $57.00 \%, \mathrm{H}, 5.03 \%, \mathrm{~N}, 13.99 \%$; found $\mathrm{C}, 56.74 \%, \mathrm{H}, 5.31 \%, \mathrm{~N}$, $14.17 \%$. 


\section{Preparation of compound 8}

To a solution of $5(3.75 \mathrm{~g}, 10.0 \mathrm{mmol})$ in absolute methanol $(70 \mathrm{ml})$ at $0^{\circ} \mathrm{C}$; thionyl chloride $(0.8$ $\mathrm{ml}, 10.0 \mathrm{mmol}$ ) was added dropwise under vigorous stirring for 3 hours at $5^{\circ} \mathrm{C}$. The reaction mixture was kept overnight at room temperature, then the solvent was evaporated under reduced pressure, afterwards the residue was treated with absolute methanol $(20 \mathrm{ml})$ with subsequent evaporation. This process was repeated three times to remove excess $\mathrm{HCl}$. The residue was chromatographed using petroleum ether/ethyl acetate $(3: 1)$ as eluent.

Methyl 1-(methyl 5-deoxy- $\beta$-D-ribofuranosyl-5-yl)-2-oxo-1,2-dihydroquinoline-4carboxylate (8). White crystals $(2.10 \mathrm{~g}, 60 \%)$; mp 187-188 ${ }^{\circ} \mathrm{C} .{ }^{1} \mathrm{H}$ NMR (250 MHz, DMSO): $\delta$ $8.85(1 \mathrm{H}, \mathrm{d}, J=8.1 \mathrm{~Hz}, \mathrm{ArH}) ; 8.0(1 \mathrm{H}, \mathrm{d}, J=8.1 \mathrm{~Hz}, \mathrm{ArH}) ; 7.81(1 \mathrm{H}, \mathrm{s}, \mathrm{CH}) ; 7.75-7.52(2 \mathrm{H}, \mathrm{m}$, ArH); 5.41-5.34 (1H, m, H-2); 5.15-5.05 (1H, m, H-3); 4.95 (1H, s, H-1); 4.66-4.58 (2H, m, H-4, $\mathrm{H}-5)$; 4.41-4.38 (1H, m, H-5 $)$; $3.62\left(3 \mathrm{H}, \mathrm{s} \mathrm{OCH}_{3}\right) ; 3.38\left(3 \mathrm{H}, \mathrm{s}, \mathrm{OCH}_{3}\right) ; 2.15(2 \mathrm{H}, \mathrm{bs}, 2 \mathrm{OH}) .{ }^{13} \mathrm{C}$ NMR (62.5 MHz, DMSO): $\delta 174.1 ; 164.6$ (2CO); 152.2; 142.8; 138.3; 131.2; 128.7; 126.5; 122.2; 115.9 (Ar-C); 107.0 (C-1); 80.3 (C-2); 76.1 (C-3); 71.3 (C-4); 66.0 (C-5); $54.0\left(\mathrm{OCH}_{3}\right)$; $53.6\left(\mathrm{OCH}_{3}\right)$. (MALDI, positive mode, matrix: DHB): $\mathrm{m} / \mathrm{z}=371.3(\mathrm{M}+\mathrm{Na})^{+}$. Anal. Calcd. For $\mathrm{C}_{17} \mathrm{H}_{19} \mathrm{NO}_{7}$ (349.34): C, $58.45 \%, \mathrm{H}, 5.48 \%, \mathrm{~N}, 4.01 \%$; found $\mathrm{C}, 58.02 \%, \mathrm{H}, 5.39 \%$, N, 3.79 $\%$.

\section{Acetylation of compound 8}

Quinoline $8(0.70 \mathrm{~g}, 2.0 \mathrm{mmol})$ was treated with acetic anhydride $(20 \mathrm{~mL})$ and pyridine $(20 \mathrm{~mL})$. The reaction mixture was stirred at room temperature for $24 \mathrm{~h}$.; concentrated under reduced pressure, and purified by flash chromatography (petroleum ether/ethyl acetate, 8:1).

Methyl 1-(methyl 5-deoxy-2,3-di- $O$-acetyl- $\beta$-D-ribofuranosyl-5-yl)-2-oxo-1,2dihydroquinoline-4-carboxylate (9). White powder $(0.60 \mathrm{~g}, 69 \%)$; mp 161-162 ${ }^{\circ} \mathrm{C} .{ }^{1} \mathrm{H}$ NMR $\left(250 \mathrm{MHz}, \mathrm{CDCl}_{3}\right): \delta$ 7.56-7.43 (2H, m, ArH); $7.29(1 \mathrm{H}, \mathrm{s}, \mathrm{CH}) ; 7.28-7.21(2 \mathrm{H}, \mathrm{m}, \mathrm{ArH}) ; 5.43-$ $5.32(1 \mathrm{H}, \mathrm{m}, \mathrm{H}-2) ; 5.25(1 \mathrm{H}, \mathrm{d}, J=5.0 \mathrm{~Hz}, \mathrm{H}-3) ; 4.91(1 \mathrm{H}, \mathrm{s}, \mathrm{H}-1)$; 4.64-4.58 (1H, m, H-4); 4.47-4.42 (2H, m, H-5, H-5 ') $3.63\left(3 \mathrm{H}, \mathrm{s}, \mathrm{OCH}_{3}\right) ; 3.36\left(3 \mathrm{H}, \mathrm{s}, \mathrm{OCH}_{3}\right) ; 2.10\left(3 \mathrm{H}, \mathrm{s}, \mathrm{CH}_{3}\right) ; 2.04$ $\left(3 \mathrm{H}, \mathrm{s}, \mathrm{CH}_{3}\right) .{ }^{13} \mathrm{C} \mathrm{NMR}\left(62.5 \mathrm{MHz}, \mathrm{CDCl}_{3}\right): \delta 174.1 ; 170.6 ; 170.1 ; 168.2(4 \mathrm{CO}) ; 153.1 ; 141.5 ;$ 139.1; 132.4; 129.2; 127.2; 124.2 (Ar-C); $116.4\left(\mathrm{CMe}_{2}\right) ; 106.5$ (C-1); 81.2 (C-2); 76.6 (C-3); 70.8 (C-4); $66.4(\mathrm{C}-5) ; 54.2\left(\mathrm{OCH}_{3}\right) ; 54.4\left(\mathrm{OCH}_{3}\right) ; 20.5 ; 20.2$ (2AcO). (MALDI, positive mode, matrix: DHB): $\mathrm{m} / \mathrm{z}=454.7(\mathrm{M}+\mathrm{Na})^{+}$. Anal. Calcd. For $\mathrm{C}_{21} \mathrm{H}_{23} \mathrm{NO}_{9}$ (433.41): $\mathrm{C}, 58.20 \%, \mathrm{H}$, $5.35 \%, \mathrm{~N}, 3.23 \%$; found C, $58.42 \%, \mathrm{H}, 5.41 \%, \mathrm{~N}, 3.52 \%$.

\section{General procedure for preparation of Compounds 10, 12a-e, 14}

To azide solution $7(4.0 \mathrm{~g}, 10.0 \mathrm{mmol})$ in ethyl acetate $(50 \mathrm{ml})$, the appropriate nucleophile [piperidine; amino acid ester hydrochloride 11a-e or proline ester hydrochloride 13 (10.0 mmol)] in ethyl acetate $(50 \mathrm{ml})$ containing $2.0 \mathrm{ml}$ of triethyl amine was added. The reaction mixture was kept at $-5^{\circ} \mathrm{C}$ for $24 \mathrm{~h}$, then at $25^{\circ} \mathrm{C}$ for another $24 \mathrm{~h}$, followed by washing with $0.5 \mathrm{~N} \mathrm{HCl}$, water, $3 \%$ solution of $\mathrm{NaHCO}_{3}$ and finally dried $\left(\mathrm{Na}_{2} \mathrm{SO}_{4}\right)$. The solution was evaporated to dryness, and the residue was recrystallized from petroleum ether/ ethyl acetate to give the desired product. 
1-(Methyl 5-deoxy-2,3- $O$-isopropylidene- $\beta$-D-ribofuranosyl-5-yl)-2-oxo-4-(piperidine-1carbonyl) -1,2-dihydroquinoline (10). White powder $(3.40 \mathrm{~g}, 77 \%)$; mp 171-172 ${ }^{\circ} \mathrm{C} .{ }^{1} \mathrm{H}$ NMR $\left(250 \mathrm{MHz}, \mathrm{CDCl}_{3}\right): \delta 7.80(1 \mathrm{H}, \mathrm{d}, J=8.3 \mathrm{~Hz}, \mathrm{ArH}) ; 7.66-7.57(2 \mathrm{H}, \mathrm{m}, \mathrm{ArH}) ; 7.38-7.32$ $(1 \mathrm{H}, \mathrm{m}, \mathrm{ArH}) ; 6.82(1 \mathrm{H}, \mathrm{s}, \mathrm{CH}) ; 5.00(1 \mathrm{H}, \mathrm{s}, \mathrm{H}-1) ; 4.80(1 \mathrm{H}, \mathrm{d}, J=6.0 \mathrm{~Hz}, \mathrm{H}-2) ; 4.63(1 \mathrm{H}, \mathrm{d}, J=$ $6.0 \mathrm{~Hz}, \mathrm{H}-3)$; 4.59-4.51 (2H, m, H-4, H-5); 4.47-4.42 (1H, m, H-5 ); $3.62\left(3 \mathrm{H}, \mathrm{s}, \mathrm{OCH}_{3}\right) ; 3.41$ $\left(4 \mathrm{H}, \mathrm{m}, 2 \mathrm{NCH}_{2}\right) ; 1.67-1.61\left(6 \mathrm{H}, \mathrm{m}, 3 \mathrm{CH}_{2}\right) ; 1.47\left(3 \mathrm{H}, \mathrm{s}, \mathrm{CH}_{3}\right) ; 1.30\left(3 \mathrm{H}, \mathrm{s}, \mathrm{CH}_{3}\right) .{ }^{13} \mathrm{C} \mathrm{NMR}$ $\left(62.5 \mathrm{MHz}, \mathrm{CDCl}_{3}\right): \delta 166.2,160.7(2 \mathrm{CO}) ; 146.5 ; 145.8 ; 130.0 ; 129.8 ; 127.6 ; 124.7 ; 121.4(\mathrm{Ar}-$ C); $112.3\left(\mathrm{CMe}_{2}\right) ; 109.3(\mathrm{CH}) ; 100.9$ (C-1); 85.1 (C-2); 84.4 (C-3); 81.9 (C-4); 66.2 (C-5); 54.7 $\left(\mathrm{OCH}_{3}\right) ; 47.9\left(\mathrm{CH}_{2}\right) ; 42.4\left(\mathrm{CH}_{2}\right) ; 33.8\left(2 \mathrm{CH}_{2}\right) ; 42.4\left(\mathrm{CH}_{2}\right) ; 26.4 ; 24.2\left(2 \mathrm{CH}_{3}\right)$. (MALDI, positive mode, matrix: DHB): $\mathrm{m} / \mathrm{z}=464.5(\mathrm{M}+\mathrm{Na})^{+}$. Anal. Calcd. For $\mathrm{C}_{24} \mathrm{H}_{30} \mathrm{~N}_{2} \mathrm{O}_{6}(442.50): \mathrm{C}, 65.14 \%$, $\mathrm{H}, 6.83 \%$, N, $6.33 \%$; found C, $65.40 \%, \mathrm{H}, 7.15 \%$, N, $6.07 \%$.

Methyl-2-\{[1-(methyl 5-deoxy-2,3- $O$-isopropylidene- $\beta$-D-ribofuranosyl-5-yl)]-2-oxo-1,2dihydroquinoline-4-carboxamido \} acetate (12a). White powder (3.60 g, $81 \%$ ); mp 148-149 ${ }^{\circ} \mathrm{C} .{ }^{1} \mathrm{H}$ NMR $\left(250 \mathrm{MHz}, \mathrm{CDCl}_{3}\right): \delta 8.05(1 \mathrm{H}, \mathrm{d}, J=7.3 \mathrm{~Hz}, \mathrm{ArH}) ; 7.77(1 \mathrm{H}, \mathrm{d}, J=7.3 \mathrm{~Hz}$, $\operatorname{ArH})$; 7.63-7.57 (1H, m, ArH); 7.41-7.32 (1H, m, ArH); $7.00(1 \mathrm{H}, \mathrm{s}, \mathrm{CH}) ; 6.78(1 \mathrm{H}, \mathrm{t}, J=5.4$ $\mathrm{Hz}, \mathrm{NH}) ; 4.99$ (1H, s, H-1); $4.79(1 \mathrm{H}, \mathrm{d}, J=6.0 \mathrm{~Hz}, \mathrm{H}-2) ; 4.61(1 \mathrm{H}, \mathrm{d}, J=6.0 \mathrm{~Hz}, \mathrm{H}-3)$; $4.57-$ $4.48(1 \mathrm{H}, \mathrm{m}, \mathrm{H}-4) ; 4.46-4.40$ (2H, m, H-5, H-5 $)$; $4.21\left(2 \mathrm{H}, \mathrm{d}, J=5.4 \mathrm{~Hz}, \mathrm{NHCH}_{2}\right) ; 3.75(3 \mathrm{H}, \mathrm{s}$, $\left.\mathrm{OCH}_{3}\right) ; 3.31\left(3 \mathrm{H}, \mathrm{s}, \mathrm{OCH}_{3}\right) ; 1.46\left(3 \mathrm{H}, \mathrm{s}, \mathrm{CH}_{3}\right) ; 1.29\left(3 \mathrm{H}, \mathrm{s}, \mathrm{CH}_{3}\right) .{ }^{13} \mathrm{C} \mathrm{NMR}(62.5 \mathrm{MHz}$, $\left.\mathrm{CDCl}_{3}\right): \delta 169.9,167.1,160.5(3 \mathrm{CO}) ; 146.9 ; 144.2 ; 130.2 ; 128.9 ; 127.7 ; 125.2 ; 121.5$ (Ar-C); $111.2\left(\mathrm{CMe}_{2}\right) ; 109.4(\mathrm{CH}) ; 107.8$ (C-1); 85.2 (C-2); 84.5 (C-3); 82.0 (C-4); 66.3 (C-5); 54.9 $\left(\mathrm{OCH}_{3}\right) ; 52.5\left(\mathrm{OCH}_{3}\right) ; 41.5\left(\mathrm{NHCH}_{2}\right) ; 26.9 ; 24.9\left(2 \mathrm{CH}_{3}\right)$. (MALDI, positive mode, matrix: DHB): $\mathrm{m} / \mathrm{z}=468.0(\mathrm{M}+\mathrm{Na})^{+}$. Anal. Calcd. For $\mathrm{C}_{22} \mathrm{H}_{26} \mathrm{~N}_{2} \mathrm{O}_{8}(446.45): \mathrm{C}, 59.19 \%$, H, $5.87 \%$, $\mathrm{N}, 6.27 \%$; found C, $58.87 \%, \mathrm{H}, 5.92 \%, \mathrm{~N}, 6.41 \%$.

Methyl-2-\{[1-(methyl 5-deoxy-2,3-O -isopropylidene- $\beta$-D-ribofuranosyl-5-yl)]-2-oxo-1,2dihydroquinoline-4-carboxamido $\}$ propanoate (12b). White powder (3.50 g, 76 \%); mp 162$163{ }^{\circ} \mathrm{C} .{ }^{1} \mathrm{H}$ NMR $\left(250 \mathrm{MHz}, \mathrm{CDCl}_{3}\right): \delta 8.11(1 \mathrm{H}, \mathrm{s}, \mathrm{CH}) ; 7.73-7.68(2 \mathrm{H}, \mathrm{m}, \mathrm{ArH}) ; 7.48-7.42$ $(1 \mathrm{H}, \mathrm{m}, \mathrm{ArH}) ; 7.18-7.11(1 \mathrm{H}, \mathrm{m}, \mathrm{ArH}) ; 6.60(1 \mathrm{H}, \mathrm{d}, J=7.6 \mathrm{~Hz}, \mathrm{NH}) ; 4.98(1 \mathrm{H}, \mathrm{s}, \mathrm{H}-1) ; 4.79$ $(1 \mathrm{H}, \mathrm{d}, J=6.0 \mathrm{~Hz}, \mathrm{H}-2) ; 4.62(1 \mathrm{H}, \mathrm{d}, J=6.0 \mathrm{~Hz}, \mathrm{H}-3) ; 4.59-4.47$ (2H, m, H-4, H-5); 4.42-4.34 $\left(2 \mathrm{H}, \mathrm{m}, \mathrm{NH} \underline{\mathrm{H}}, \mathrm{H}-5^{\prime}\right) ; 3.68\left(3 \mathrm{H}, \mathrm{s}, \mathrm{OCH}_{3}\right) ; 3.30\left(3 \mathrm{H}, \mathrm{s}, \mathrm{OCH}_{3}\right) ; 1.46\left(3 \mathrm{H}, \mathrm{s}, \mathrm{CH}_{3}\right) ; 1.40(3 \mathrm{H}, \mathrm{d}$, $\left.J=7.2 \mathrm{~Hz}, \mathrm{CH}_{3}\right) ; 1.29\left(3 \mathrm{H}, \mathrm{s}, \mathrm{CH}_{3}\right) .{ }^{13} \mathrm{C} \mathrm{NMR}\left(62.5 \mathrm{MHz}, \mathrm{CDCl}_{3}\right): \delta 174.8 ; 162.3 ; 154.7$ (3CO); 146.9; 143.7; 131.4; 129.4; 128.0; 123.5; $119.9(\mathrm{Ar}-\mathrm{C}) ; 112.3\left(\mathrm{CMe}_{2}\right) ; 109.4(\mathrm{CH})$; 100.7 (C-1); 85.3 (C-2); 84.5 (C-3); 82.1 (C-4); 65.7 (C-5); $54.8\left(\mathrm{OCH}_{3}\right) ; 52.6\left(\mathrm{OCH}_{3}\right) ; 48.9$ $(\mathrm{NHCH}), 26.4 ; 24.9\left(2 \mathrm{CH}_{3}\right) ; 18.6\left(\mathrm{CH}_{3}\right)$. (MALDI, positive mode, matrix: DHB): $\mathrm{m} / \mathrm{z}=482.6$ $(\mathrm{M}+\mathrm{Na})^{+}$. Anal. Calcd. For $\mathrm{C}_{23} \mathrm{H}_{28} \mathrm{~N}_{2} \mathrm{O}_{8}$ (460.48): C, $59.99 \%, \mathrm{H}, 6.13 \%, \mathrm{~N}, 6.08 \%$; found C, $60.12 \%, \mathrm{H}, 5.95 \%$, N, $6.41 \%$.

Methyl-2-\{[1-(methyl 5-deoxy-2,3-O-isopropylidene- $\beta$-D-ribofuranosyl-5-yl)]-2-oxo-1,2dihydroquinoline-4-carboxamido 3 3-methylbutanoate (12c). White powder $(3.10 \mathrm{~g}, 63 \%)$; mp 129-130 ${ }^{\circ} \mathrm{C} .{ }^{1} \mathrm{H}$ NMR $\left(250 \mathrm{MHz}, \mathrm{CDCl}_{3}\right): \delta$ 7.78-7.65 (2H, m, ArH); $7.52(1 \mathrm{H}, \mathrm{s}, \mathrm{CH})$; 7.47-7.41 (2H, m, ArH); $6.68(1 \mathrm{H}, \mathrm{d}, J=8.5 \mathrm{~Hz}, \mathrm{NH}) ; 4.96(1 \mathrm{H}, \mathrm{s}, \mathrm{H}-1) ; 4.78(1 \mathrm{H}, \mathrm{d}, J=6.1 \mathrm{~Hz}$, H-2); 4.59 (1H, d, $J=6.1 \mathrm{~Hz}, \mathrm{H}-3)$; 4.56-4.48 (2H, m, H-4, H-5); 4.43-4.34 (2H, m, H-5 ', 
$\mathrm{NHCH}) ; 3.63\left(3 \mathrm{H}, \mathrm{s}, \mathrm{OCH}_{3}\right) ; 3.28\left(3 \mathrm{H}, \mathrm{s}, \mathrm{OCH}_{3}\right) ; 2.14-2.03(1 \mathrm{H}, \mathrm{m}, \mathrm{CH}) ; 1.45\left(3 \mathrm{H}, \mathrm{s}, \mathrm{CH}_{3}\right)$; $1.28\left(3 \mathrm{H}, \mathrm{s}, \mathrm{CH}_{3}\right) ; 0.93\left(3 \mathrm{H}, \mathrm{d}, J=6.8 \mathrm{~Hz}, \mathrm{CH}_{3}\right) ; 0.83\left(3 \mathrm{H}, \mathrm{d}, J=6.8 \mathrm{~Hz}, \mathrm{CH}_{3}\right) .{ }^{13} \mathrm{C} \mathrm{NMR}(62.5$ $\left.\mathrm{MHz}, \mathrm{CDCl}_{3}\right): \delta 173.8 ; 162.5 ; 155.4(3 \mathrm{CO}) ; 146.9 ; 144.1 ; 133.4 ; 129.5 ; 128.0 ; 123.5 ; 120.2(\mathrm{Ar}-$ C); $111.1\left(\mathrm{CMe}_{2}\right) ; 109.5(\mathrm{CH}) ; 100.5(\mathrm{C}-1) ; 85.4$ (C-2); 84.6 (C-3); 82.2 (C-4); 65.8 (C-5); 58.4 $(\mathrm{NHCH}) ; 54.9\left(\mathrm{OCH}_{3}\right) ; 52.3\left(\mathrm{OCH}_{3}\right) ; 31.3(\mathrm{CH}) ; 26.5,25.0\left(2 \mathrm{CH}_{3}\right) ; 19.1 ; 17.9\left(2 \mathrm{CH}_{3}\right)$. (MALDI, positive mode, matrix: DHB): $\mathrm{m} / \mathrm{z}=511.0(\mathrm{M}+\mathrm{Na})^{+} ; 526.0(\mathrm{M}+\mathrm{K})^{+}$. Anal. Calcd. For $\mathrm{C}_{25} \mathrm{H}_{32} \mathrm{~N}_{2} \mathrm{O}_{8}$ (488.53): C, $61.46 \%, \mathrm{H}, 6.60 \%, \mathrm{~N}, 5.73 \%$; found $\mathrm{C}, 61.12 \%, \mathrm{H}, 6.43 \%, \mathrm{~N}, 5.86$ $\%$.

Methyl-2-\{[1-(methyl 5-deoxy-2,3- $O$-isopropylidene- $\beta$-D-ribofuranosyl-5-yl)]-2-oxo-1,2dihydroquinoline-4-carboxamido 4-methylpentanoate (12d). White powder (3.70 g, 73 \%); mp 107-108 ${ }^{\circ} \mathrm{C} .{ }^{1} \mathrm{H}$ NMR $\left(250 \mathrm{MHz}, \mathrm{CDCl}_{3}\right): \delta$ 8.00-7.76 $(1 \mathrm{H}, \mathrm{m}, \mathrm{ArH}) ; 7.73-7.69(2 \mathrm{H}, \mathrm{m}$, ArH); 7.51-7.45 (1H, m, ArH); $7.42(1 \mathrm{H}, \mathrm{s}, \mathrm{CH}) ; 6.46(1 \mathrm{H}, \mathrm{d}, J=8.1 \mathrm{~Hz}, \mathrm{NH}) ; 4.98(1 \mathrm{H}, \mathrm{s}, \mathrm{H}-$ 1); $4.77(1 \mathrm{H}, \mathrm{d}, J=5.9 \mathrm{~Hz}, \mathrm{H}-2) ; 4.59(1 \mathrm{H}, \mathrm{d}, J=5.9 \mathrm{~Hz}, \mathrm{H}-3)$; 4.55-4.44 (2H, m, H-4, H-5); 4.41-4.33 (2H, m, H-5 , NHH $) ; 3.73\left(3 \mathrm{H}, \mathrm{s}, \mathrm{OCH}_{3}\right) ; 3.30\left(3 \mathrm{H}, \mathrm{s}, \mathrm{OCH}_{3}\right) ; 1.79-1.56(2 \mathrm{H}, \mathrm{m}$, $\left.\mathrm{CH}_{2}\right) ; 1.47\left(3 \mathrm{H}, \mathrm{s}, \mathrm{CH}_{3}\right) ; 1.29\left(3 \mathrm{H}, \mathrm{s}, \mathrm{CH}_{3}\right) ; 1.26-1.22(1 \mathrm{H}, \mathrm{m}, \mathrm{CH}) ; 3.93(6 \mathrm{H}, 2 \mathrm{~d}, J=3.8 \mathrm{~Hz}$, $\left.2 \mathrm{CH}_{3}\right) .{ }^{13} \mathrm{C} \mathrm{NMR}\left(62.5 \mathrm{MHz}, \mathrm{CDCl}_{3}\right): \delta 175.6 ; 162.2 ; 154.7(3 \mathrm{CO}) ; 146.7 ; 143.4 ; 130.0 ; 129.2 ;$ 123.3; 119.6; 117.9 (Ar-C); $112.2\left(\mathrm{CMe}_{2}\right) ; 109.3(\mathrm{CH}) ; 100.3$ (C-1); 85.1 (C-2); 84.4 (C-3); 82.0 $(\mathrm{C}-4) ; 65.5(\mathrm{C}-5) ; 54.7\left(\mathrm{OCH}_{3}\right) ; 52.5\left(\mathrm{OCH}_{3}\right) ; 51.6(\mathrm{NHCH}) ; 41.4(\mathrm{CH}) ; 26.3\left(\mathrm{CH}_{3}\right) ; 25.0$ $\left(\mathrm{CH}_{2}\right) ; 24.8\left(\mathrm{CH}_{3}\right) ; 22.6\left(\mathrm{CH}_{3}\right) ; 21.7\left(\mathrm{CH}_{3}\right)$. (MALDI, positive mode, matrix: DHB): $\mathrm{m} / \mathrm{z}=525.7$ $(\mathrm{M}+\mathrm{Na})^{+} ; 540.6(\mathrm{M}+\mathrm{K})^{+}$. Anal. Calcd. For $\mathrm{C}_{26} \mathrm{H}_{34} \mathrm{~N}_{2} \mathrm{O}_{8}$ (502.56): C, $62.14 \%$, H, $6.82 \%$, N, 5.57 $\%$; found $\mathrm{C}, 62.43 \%, \mathrm{H}, 7.10 \%, \mathrm{~N}, 5.41 \%$.

Methyl-2-\{[1-(methyl 5-deoxy-2,3-O -isopropylidene- $\beta$-D-ribofuranosyl-5-yl)]-2-oxo-1,2dihydroquinoline-4-carboxamido 4-methylsulfanylbutanoate (12e). White powder (2.80 g, $54 \%$ ); mp 81-82 ${ }^{\circ} \mathrm{C} .{ }^{1} \mathrm{H}$ NMR $\left(250 \mathrm{MHz}, \mathrm{CDCl}_{3}\right): \delta 8.12(1 \mathrm{H}, \mathrm{s}, \mathrm{CH}) ; 7.77-7.71(2 \mathrm{H}, \mathrm{m}, \mathrm{ArH})$; 7.55-7.47 (1H, m, ArH); 7.25-7.17 (1H, m, ArH); $6.68(1 \mathrm{H}, \mathrm{d}, J=8.0 \mathrm{~Hz}, \mathrm{NH}) ; 4.99(1 \mathrm{H}, \mathrm{s}, \mathrm{H}-$ 1); $4.80(1 \mathrm{H}, \mathrm{d}, J=6.0 \mathrm{~Hz}, \mathrm{H}-2)$; $4.62(1 \mathrm{H}, \mathrm{d}, J=6.0 \mathrm{~Hz}, \mathrm{H}-3) ; 4.58-4.50$ (2H, m, H-4, H-5); 4.46-4.33 (2H, m, H-5 ' NHCH $) ; 3.69\left(3 \mathrm{H}, \mathrm{s}, \mathrm{OCH}_{3}\right) ; 3.32\left(3 \mathrm{H}, \mathrm{s}, \mathrm{OCH}_{3}\right) ; 2.51(1 \mathrm{H}, \mathrm{t}, J=7.3$ $\left.\mathrm{Hz}, \mathrm{CH}_{2}\right) ; 2.18-2.05\left(2 \mathrm{H}, \mathrm{m}, \underline{\mathrm{CH}}_{2} \mathrm{~S}\right) ; 2.01\left(3 \mathrm{H}, \mathrm{s}, \mathrm{SCH}_{3}\right) ; 1.47\left(3 \mathrm{H}, \mathrm{s}, \mathrm{CH}_{3}\right) ; 1.30\left(3 \mathrm{H}, \mathrm{s}, \mathrm{CH}_{3}\right)$. ${ }^{13} \mathrm{C} \mathrm{NMR}\left(62.5 \mathrm{MHz}, \mathrm{CDCl}_{3}\right): \delta 173.7,162.3,154.8(3 \mathrm{CO}) ; 146.9 ; 143.6 ; 133.5 ; 129.5 ; 128.0$; 123.5; 119.9 (Ar-C); $112.3\left(\mathrm{CMe}_{2}\right) ; 109.4(\mathrm{CH}) ; 100.9$ (C-1); 85.2 (C-2); 84.4 (C-3); 82.1 (C-4); $65.7(\mathrm{C}-5) ; 54.6(\mathrm{NHCH}) ; 52.6\left(\mathrm{OCH}_{3}\right) ; 52.4\left(\mathrm{OCH}_{3}\right) ; 31.7\left(\mathrm{CH}_{2}\right) ; 29.9\left(\mathrm{CH}_{2}\right) ; 26.4\left(\mathrm{CH}_{3}\right) ; 24.9$ $\left(\mathrm{CH}_{3}\right) ; 15.3\left(\mathrm{SCH}_{3}\right)$. (MALDI, positive mode, matrix: DHB): $\mathrm{m} / \mathrm{z}=542.5(\mathrm{M}+\mathrm{Na})^{+}$. Anal. Calcd. For $\mathrm{C}_{25} \mathrm{H}_{32} \mathrm{~N}_{2} \mathrm{O}_{8} \mathrm{~S}$ (520.60): C, $57.68 \%, \mathrm{H}, 6.20 \%$, N, $5.38 \%$; found C, $57.52 \%, \mathrm{H}, 5.95 \%$, N, $5.21 \%$.

Benzyl 1-\{[1-(methyl 5-deoxy-2,3-O-isopropylidene- $\beta$-D-ribofuranosyl-5-yl)]-2-oxo-1,2dihydroquinoline- 4-carbonyl pyrrolidin-2-carboxylate (14). White powder (3.80 g, 67 \%); mp 68-69 ${ }^{\circ} \mathrm{C} .{ }^{1} \mathrm{H}$ NMR $\left(250 \mathrm{MHz}, \mathrm{CDCl}_{3}\right): \delta 7.80(1 \mathrm{H}, \mathrm{d}, J=8.4 \mathrm{~Hz}, \mathrm{ArH}) ; 7.71(1 \mathrm{H}, \mathrm{s}, \mathrm{CH})$; 7.61-7.54 (4H, m, ArH); 7.34-7.28 (4H, m, ArH); $5.28\left(1 \mathrm{H}, \mathrm{d}, J_{\text {gem }}=12.3 \mathrm{~Hz}, \mathrm{CHph}\right) ; 5.21(1 \mathrm{H}$, $\left.\mathrm{d}, J_{\text {gem }}=12.3 \mathrm{~Hz}, \mathrm{CHph}\right) ; 5.01(1 \mathrm{H}, \mathrm{s}, \mathrm{H}-1) ; 4.83(1 \mathrm{H}, \mathrm{d}, J=6.0 \mathrm{~Hz}, \mathrm{H}-2) ; 4.63(1 \mathrm{H}, \mathrm{d}, J=6.0$ $\mathrm{Hz}, \mathrm{H}-3)$; 4.62-4.51 (2H, m, H-4, H-5); 4.44-4.36 (2H, m, H-5 ' NHCH$) ; 3.86-3.60$ (2H, m, 
$\left.\mathrm{CH}_{2}\right) ; 3.34\left(3 \mathrm{H}, \mathrm{s}, \mathrm{OCH}_{3}\right) ; 2.23-2.01\left(4 \mathrm{H}, \mathrm{m}, 2 \mathrm{CH}_{2}\right) ; 1.48\left(3 \mathrm{H}, \mathrm{s}, \mathrm{CH}_{3}\right) ; 1.31\left(3 \mathrm{H}, \mathrm{s}, \mathrm{CH}_{3}\right) .{ }^{13} \mathrm{C}$ NMR $\left(62.5 \mathrm{MHz}, \mathrm{CDCl}_{3}\right): \delta 177.7 ; 172.4 ; 162.6(3 \mathrm{CO}) ; 153.2 ; 146.9 ; 143.3 ; 135.2 ; 129.3$; 128.6; 128.5; 128.2; 123.6; 118.9; 117.9 (Ar-C); $112.3\left(\mathrm{CMe}_{2}\right) ; 109.4(\mathrm{CH}) ; 100.3(\mathrm{C}-1) ; 85.3$ (C-2); 84.5 (C-3); 82.1 (C-4); $67.4(\mathrm{C}-5) ; 65.7\left(\underline{\mathrm{CH}}_{2} \mathrm{Ph}\right) ; 59.6(\mathrm{NCH}) ; 54.8\left(\mathrm{OCH}_{3}\right) ; 46.6\left(\mathrm{CH}_{2}\right)$; $29.5\left(\mathrm{CH}_{2}\right) ; 26.4\left(\mathrm{CH}_{3}\right) ; 24.9\left(\mathrm{CH}_{3}\right) ; 24.3\left(\mathrm{CH}_{2}\right)$. (MALDI, positive mode, matrix: DHB): $\mathrm{m} / \mathrm{z}=$ $584.4(\mathrm{M}+\mathrm{Na})^{+}$. Anal. Calcd. For $\mathrm{C}_{31} \mathrm{H}_{34} \mathrm{~N}_{2} \mathrm{O}_{8}(562.23)$ : C, $66.18 \%, \mathrm{H}, 6.09 \%, \mathrm{~N}, 4.98 \%$; found $\mathrm{C}, 65.92 \%, \mathrm{H}, 5.85 \%, \mathrm{~N}, 5.26 \%$.

\section{General procedure for preparation of hydrazide 15}

The hydrazinolysis of 12a was processed as described above and the product 15 was purified by crystalisation from ethanol.

2-\{[1-(methyl 5-deoxy-2,3-O-isopropylidene- $\beta$-D-ribofuranosyl-5-yl)]-2-oxo-1,2-dihydroquinoline-4-carboxamido\} acetohydrazide (15). White powder (3.20 g, $72 \%)$; mp 101-103 ${ }^{\circ} \mathrm{C} .{ }^{1} \mathrm{H}$ NMR $\left(250 \mathrm{MHz}, \mathrm{CDCl}_{3}\right): \delta 8.23(1 \mathrm{H}, \mathrm{d}, J=7.5 \mathrm{~Hz}, \mathrm{ArH}) ; 7.79(2 \mathrm{H}, \mathrm{d}, J=7.5 \mathrm{~Hz}$, ArH); 7.71-7.59 (2H, m, ArH); $7.01(1 \mathrm{H}, \mathrm{s}, \mathrm{CH}) ; 6.85(1 \mathrm{H}, \mathrm{bs}, \mathrm{NH}) ; 5.05(1 \mathrm{H}, \mathrm{s}, \mathrm{H}-1)$; 4.61$4.54(2 \mathrm{H}, \mathrm{m}, \mathrm{H}-2, \mathrm{H}-3)$; 4.50-4.43 (3H, m, H-4, H-5, H-5 ); 4.13 (2H, d, J = 5.7 Hz, NHCH $3.85\left(3 \mathrm{H}, \mathrm{s}, \mathrm{OCH}_{3}\right) ; 3.50\left(2 \mathrm{H}, \mathrm{bs}, \mathrm{NH}_{2}\right) ; 1.47\left(3 \mathrm{H}, \mathrm{s}, \mathrm{CH}_{3}\right) ; 1.21\left(3 \mathrm{H}, \mathrm{s}, \mathrm{CH}_{3}\right)$. (MALDI, positive mode, matrix: DHB): $\mathrm{m} / \mathrm{z}=468.3(\mathrm{M}+\mathrm{Na})^{+}$. Anal. Calcd. For $\mathrm{C}_{21} \mathrm{H}_{26} \mathrm{~N}_{4} \mathrm{O}_{7}$ (446.45): C, $56.50 \%$, $\mathrm{H}, 5.87 \%, \mathrm{~N}, 12.55 \%$; found $\mathrm{C}, 56.72 \%, \mathrm{H}, 5.98 \%, \mathrm{~N}, 12$.

\section{General procedure for preparation of dipeptide 16}

The azide coupling was processed as described above and the product $\mathbf{1 6}$ was purified by crystalisation from petroleum ether/ethyl acetate.

Methyl-2-\{\{[1-(methyl 5-deoxy-2,3- $O$-isopropylidene- $\beta$-D-ribofuranosyl-5-yl)]-2-oxo-1,2dihydroquinoline-4-carboxamido\}-2 acetamido\} acetate (16). White powder (2.80 g, $56 \%$ ); mp 144-145 ${ }^{\circ} \mathrm{C} .{ }^{1} \mathrm{H}$ NMR $\left(250 \mathrm{MHz}, \mathrm{CDCl}_{3}\right): \delta 8.28(1 \mathrm{H}, \mathrm{d}, J=8.3 \mathrm{~Hz}, \mathrm{ArH}) ; 8.00(1 \mathrm{H}, \mathrm{d}, J=$ $8.3 \mathrm{~Hz}, \mathrm{ArH}) ; 7.84(1 \mathrm{H}, \mathrm{dd}, J=1.4,8.3 \mathrm{~Hz}, \mathrm{ArH}) ; 7.60(1 \mathrm{H}, \mathrm{dd}, J=1.4,8.3 \mathrm{~Hz}, \mathrm{ArH}) ; 7.31$ $(1 \mathrm{H}, \mathrm{bs}, \mathrm{NH}) ; 7.24(1 \mathrm{H}, \mathrm{s}, \mathrm{CH}) ; 7.03(1 \mathrm{H}, \mathrm{bs}, \mathrm{NH}) ; 5.21(1 \mathrm{H}, \mathrm{s}, \mathrm{H}-1) ; 5.00(1 \mathrm{H}, \mathrm{d}, J=6.0 \mathrm{~Hz}, \mathrm{H}-$ 2); $4.82(1 \mathrm{H}, \mathrm{d}, J=6.0 \mathrm{~Hz}, \mathrm{H}-3) ; 4.78-4.64(3 \mathrm{H}, \mathrm{m}, \mathrm{H}-4, \mathrm{H}-5, \mathrm{H}-5)$ ); $4.43(2 \mathrm{H}, \mathrm{d}, J=5.3 \mathrm{~Hz}$, $\left.\mathrm{NHCH}_{2}\right) ; 4.24\left(2 \mathrm{H}, \mathrm{d}, J=5.3 \mathrm{~Hz}, \mathrm{NHCH}_{2}\right) ; 3.92\left(3 \mathrm{H}, \mathrm{s}, \mathrm{OCH}_{3}\right) ; 3.52\left(3 \mathrm{H}, \mathrm{s}, \mathrm{OCH}_{3}\right) ; 1.66(3 \mathrm{H}$, $\left.\mathrm{s}, \mathrm{CH}_{3}\right) ; 1.50\left(3 \mathrm{H}, \mathrm{s}, \mathrm{CH}_{3}\right) .{ }^{13} \mathrm{C}$ NMR $\left(62.5 \mathrm{MHz}, \mathrm{CDCl}_{3}\right): \delta 170.0 ; 168.6 ; 167.5 ; 160.6(4 \mathrm{CO})$; $147.1 ; 144.2 ; 135.3 ; 130.3 ; 127.8 ; 125.3 ; 121.6(\mathrm{Ar}-\mathrm{C}) ; 111.5\left(\mathrm{CMe}_{2}\right) ; 109.5(\mathrm{CH}) ; 100.7(\mathrm{C}-1)$; 85.4 (C-2); 84.6 (C-3); 82.2 (C-4); $66.4(\mathrm{C}-5) ; 55.0\left(\mathrm{OCH}_{3}\right) ; 52.5\left(\mathrm{OCH}_{3}\right) ; 43.3,41.3\left(2 \mathrm{NHCH}_{2}\right)$, 26.5; $25.1\left(2 \mathrm{CH}_{3}\right)$. (MALDI, positive mode, matrix: DHB): $\mathrm{m} / \mathrm{z}=525.5(\mathrm{M}+\mathrm{Na})^{+}$. ). Anal. Calcd. For $\mathrm{C}_{24} \mathrm{H}_{29} \mathrm{~N}_{3} \mathrm{O}_{9}$ (503.50): C, $57.25 \%, \mathrm{H}, 5.81 \%$, N, $8.35 \%$; found C, $56.93 \%, \mathrm{H}, 6.04$ $\%, \mathrm{~N}, 8.12 \%$. 


\section{References}

1. Balasubramanian, M.; Keay, J. G. In Katritzky, A. R.; Rees, C. W.; Scriven, E. F. V., Eds., Comprehensive Heterocyclic Chemistry II; Vol. 5, Pergamon: Oxford, 1996.

2. De, D.; Byers, L. D.; Krogstad, D. J. J. Heterocycl. Chem. 1997, 34, 315.

3. Gilchrist, T. J. Chem. Soc., Perkin Trans. 1 2001, 2491.

4. Kouznetsov, V.; Mendez, L.; Gomes, C. Curr. Org. Chem. 2005, 9, 141.

5. Elderfield, R. C.; Le Von, E. F. J. Org. Chem. 1960, 25, 1576.

6. Musiol, R.; Jampilek, J.; Buchta, V.; Silva, L.; Niedbala, H.; Podeszwa, B.; Palka, A.; Majerz-Maniecka, K.; Oleksyn, B.; Polanski, J. Bioorg. Med. Chem. 2006, 14, 3592.

7. Bénard, C.; Zouhiri, F.; Normand-Bayle, M.; Danet, M.; Desmaële, D.; Leh, H.; Mouscadet, J-F.; Mbemba, G.; Thomas, C-M.; Bonnenfant, S.; Le Bret, M.; d'Angelo, J. Bioorg. Med. Chem. Lett. 2004, 14, 2473.

8. Garrouste, P.; Pawlowski, M. Tonnaire T.; Sicsic, S.; Dumy, P.; De Rosny, E.; ReboudRavaux, M.; Fulcrand, P.; Martinez, J. Eur. J. Med. Chem. 1998, 33, 423.

9. Desrivot, J.; Herrenknecht, C.; Ponchel, G.; Garbi, N.; Prina, E.; Fournet, A.; Bories, C.; Figadére, B.; Hocquemiller, R.; Loiseau, P. M. Biomed. Pharmacother. 2007, 61, 441.

10. Borioni, A.; Mustazza, C.; Sestili, I.; Sbraccia, M.; Turchetto, L.; Del Giudice, M. F. Arch. Pharm. 2007, 340, 17.

11. Sprecher, A.; Gerspacher, M.; Beck, A.; Kimmel, S.; Wiestner, H.; Anderson, G. P.; Niederhauser, U.; Subramanian, N.; Bray, M. A. Bioorg. Med. Chem. Lett. 1998, 8, 965.

12. Galemmo, R. A.; Gavai, A.; Huang, F-C. Curr. Opin. Ther. Patents 1992, 811.

13. Musser, J. H.; Kreft, A. F. Drugs Fut. 1990, 15, 73.

14. Miescher, K. Helv. Chim. Acta 1932, 15, 163.

15. Billah, M.; Buckley, G. M.; Cooper, N.; Dyke, H. J.; Egan, R.; Ganguly, A.; Gowers, L.; Haughan, A. F.; Kendall, H. J.; Lowe, C.; Minnicozzi, M.; Montana, J. G.; Oxford, J.; Peake, J. C.; Picken, C. L.; Piwinski, J. J.; Naylor, R.; Sabin, V.; Shih, N.-Y.; Warneck, J. B. H. Bioorg. Med. Chem. Lett. 2002, 12, 1617.

16. Zhang, D; Bender, D. M.; Victor, F.; Peterson, J. A.; Boyer, R. D.;Stephenson, G. A.; Azman, A.; McCarthy, J. R. Tetrahedron Lett. 2008, 49, 2052.

17. 17.Threlfall, R.; Davies, A.; Howarth, N.; Richard Cosstick, R. Nucleosides, Nucleotides \& Nucleic acids 2007, 26, 611.

18. Knapp, S. Chem. Rev. 1995, 95, 1859. (b) Casiraghi, G.; Zanardi, F.; Rassu, G.; Spanu, P. Chem. Rev. 1995, 95, 1677.

19. Ali, I. A. I.; Al-Masoudi, I. A.; Saeed, B.; Al-Masoudi, N. A.; La Colla, P. Heteroatom Chem. 2005, 16, 148.

20. Fathalla, W.; Ali, I. A. I. Heteroatom Chem. 2007, 18, 637.

21. Fathalla, W.; El Rayes, S. M.; Ali, I. A. I. ARKIVOC 2007, (xvi), 173.

22. El Rayes, S. M.; Ali, I. A. I.; Fathalla, W. ARKIVOC 2008, (xi), 86.

23. Ali, I. A. I.; Fathalla, W.; El Rayes, S. M. ARKIVOC 2008, (iii), 179. 
24. Okada, K.; Sakuma, H.; Kondo, M.; Inoue, S. Chem. Lett. 1979, 213.

25. Abdel-Rahman, A. A.-H.; Abdel-Megied, A. E.-S.; Goda, A. E.-S.; Zeid, I. F.; El Ashry, E. S. H. Nucleosides, Nucleotides \& Nucleic acids 2003, 22, 2027.

26. Kissman, H. .M.; Baker, B. R. J. Am. Chem. Soc. 1957, 79, 5534.

27. Ghose, A. K.; Viswanadhan, V. N.; Wendoloski, J. J. Comb. Chem. 1999, 55.

28. Sahin, G.; Palaska, E.; Ekizoglu, M.; Ozalp, M. Farmaco 2002, 57, 539. 